\title{
Serial Version Unique Identifier
}

National Cancer Institute

\section{Source}

National Cancer Institute. Serial Version Unique Identifier. NCI Thesaurus. Code C42735.

Maintains a reference (via a proxy to the EVSManager). 